\title{
Use of vaginal mesh; an Asian perspective footnote from the pan-Asia meeting
}

\author{
Tsia-Shu Lo ${ }^{1,2,3,4,5}$. Yiap Loong Tan ${ }^{6}$ \\ Accepted: 24 December 2019 /Published online: 13 January 2020 \\ (C) The International Urogynecological Association 2020
}

Since the 1950s, surgical mesh has been used to repair abdominal wall hernias. In the 1970s, gynecologic surgeons began using it via an abdominal approach to correct pelvic organ prolapse (POP). Finally, in the 1990s, gynecologic surgeons began the widespread use of mesh for the surgical treatment of stress urinary incontinence (SUI) and transvaginal repair of POP [1]. Over the years, the presumed increased in demand for these types of surgeries prompted the development and manufacturing of mesh products and subsequently mesh 'kits.'

The usage and attitude toward transvaginal mesh (TVM) are globally very diverse (Fig.1). In Asia, Taiwan [2], Japan and Singapore were early adopters of transvaginal mesh in surgery to correct POP. Within a few years other countries such as China, Hong Kong, Malaysia and Thailand became adopters as transvaginal mesh use became widespread throughout Asia. In the Pan-Asia Meeting of the International Urogynecological Association Meeting 2017, a

Tsia-Shu Lo

2378@ cgmh.org.tw

1 Division of Urogynecology, Department of Obstetrics and Gynecology, Linkou, Chang Gung Memorial Hospital, Linkou Medical Center, 5, Fu-Hsin Street, Kwei-shan, Tao-Yuan City, Taiwan 333, Republic of China

2 Department of Obstetrics and Gynecology, Chang Gung Memorial Hospital, Keelung Medical Center, Keelung, Taiwan, Republic of China

3 Department of Obstetrics and Gynecology, Chang Gung Memorial Hospital, Taipei Medical Center, Taipei, Taiwan, Republic of China

4 Chang Gung University, School of Medicine, Taoyuan, Taiwan, Republic of China

5 The Asia International Advisory Board (IAB) Representative (20152018), International Urogynecology Association (IUGA), Burnsville, Minnesota, USA

6 Department of Obstetrics and Gynecology, Kuching Specialist Hospital, KPJ, Healthcare Group, Kuching, Sarawak, Malaysia survey carried out in 11 selected countries showed that gynecologists/urogynecologists from China (40\%), Taiwan (68\%) and Japan (47.4\%), and Hong Kong (25\%) used mesh more than native tissue for primary POP stages III and IV (Fig. 2). While transvaginal mesh was the route of choice, the latter two (Japan and Hong Kong) were more inclined to perform laparoscopic colpopexy. Most urogynecologists from the Southeast Asian region were more likely to use native tissue repairs for primary surgery, except for Singapore. For recurrent advanced prolapse, all of the Asian countries surveyed favored the use of mesh (Fig. 3). One reason for the difference in uptake of transvaginal mesh procedures for primary POP procedures in Southeast Asia could be that mesh kits were introduced later to the nations where the field of urogynecology was still in its infancy.

In recent years, the FDA has issued health warnings about complications of transvaginal mesh used to correct POP. Earlier this year, manufacturers were ordered to stop selling and distributing surgical mesh intended for transvaginal repair of POP immediately in the US. This was preceded by lawsuits and litigation pertaining to or related to the complications from vaginal mesh surgery. Meanwhile, in Asia, from our local experience, these complications are not as frequently reported or documented. Perhaps surgeons performing transvaginal mesh procedures are more uniformly specialized in the field of urogynecology and have received the appropriate training. Hence, the incidence of mesh complications may be lower and, if such complications occur, dealt with appropriately. Many general gynecologists may not consider offering these forms of surgery and would refer to a urogynecologist or refer to a center with the available expertise.

The subspeciality of urogynecology developed at different rates throughout Asia, which may be a reason for the various attitudes toward transvaginal mesh for POP. The field developed earlier in Asian countries such as Taiwan, Japan and Singapore where the use of transvaginal mesh is more accepted, particularly for 
Fig. 1 Current use of mesh for POP (2018)

primary treatment. Due to the negative worldwide perception of transvaginal mesh for POP, newly trained urogynecologists may be hesitant to move toward this type of mesh surgery, especially in recent years as complications are becoming more apparent and more often reported. This has led to those countries doing few transvaginal mesh repairs and often sending their fellows to neighboring countries for training with those urogynecologists doing high-volume transvaginal mesh

\section{Current use of mesh for POP}

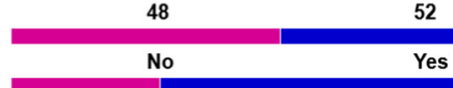

Southerm/Mediterranean Europe (ttaly, Spain, France, etc.)

Ooearia(Australa, NewZealand, etc.)

Northem Europe (British Isles, Scandinavia, Holland, Germary, etc

NorthAmerica(US, Canaca)

Latin America (nduding Mexico and Carbbean)

Indaand west Asia(induding MiddeEast)

EastemEurope

EastAsia (Japan, Korea, Singapore, Tawan, etc)

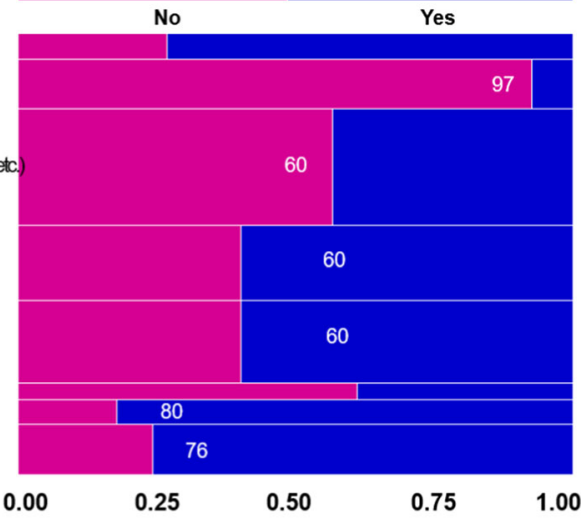

Davila GW. IUGA annual meeting. June $30^{\text {th }}, 2018$ repairs for POP to obtain the necessary knowledge and skills from the more experienced colleagues.

Patient outcomes and complication rates for mesh surgery are being extensively studied and reported in more and more Asian countries. The concept of 'out of sight, out of mind' regarding mesh complications is becoming the exception as the close and constant monitoring of patients who have had this type of surgery is more commonplace. Long-term followup of these cohorts of patients is necessary for us to better

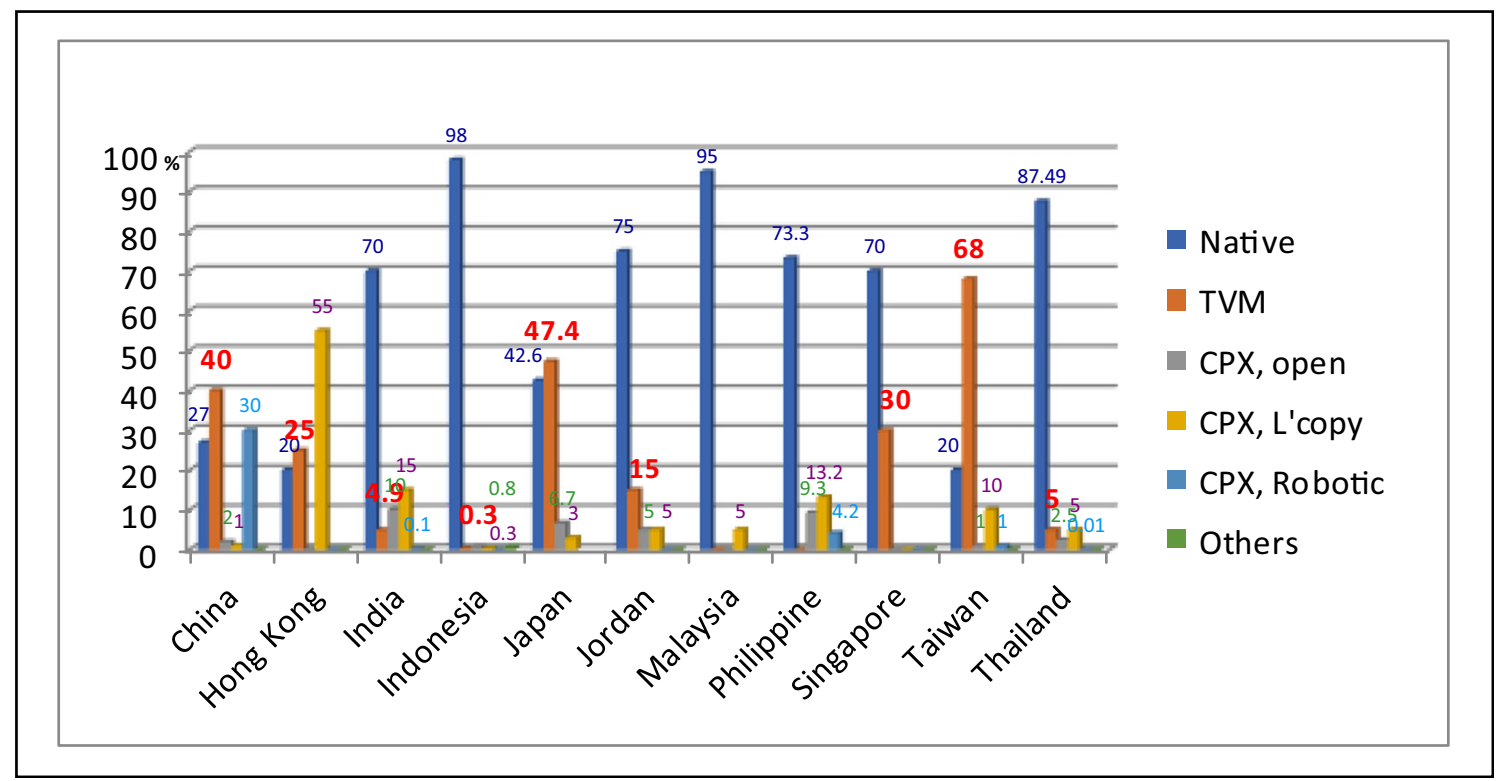

Fig. 2 Type of PRS (pelvic reconstructive surgery) for primary POP (POPQ III and IV) in 11 selected countries/areas, Asia 2018 


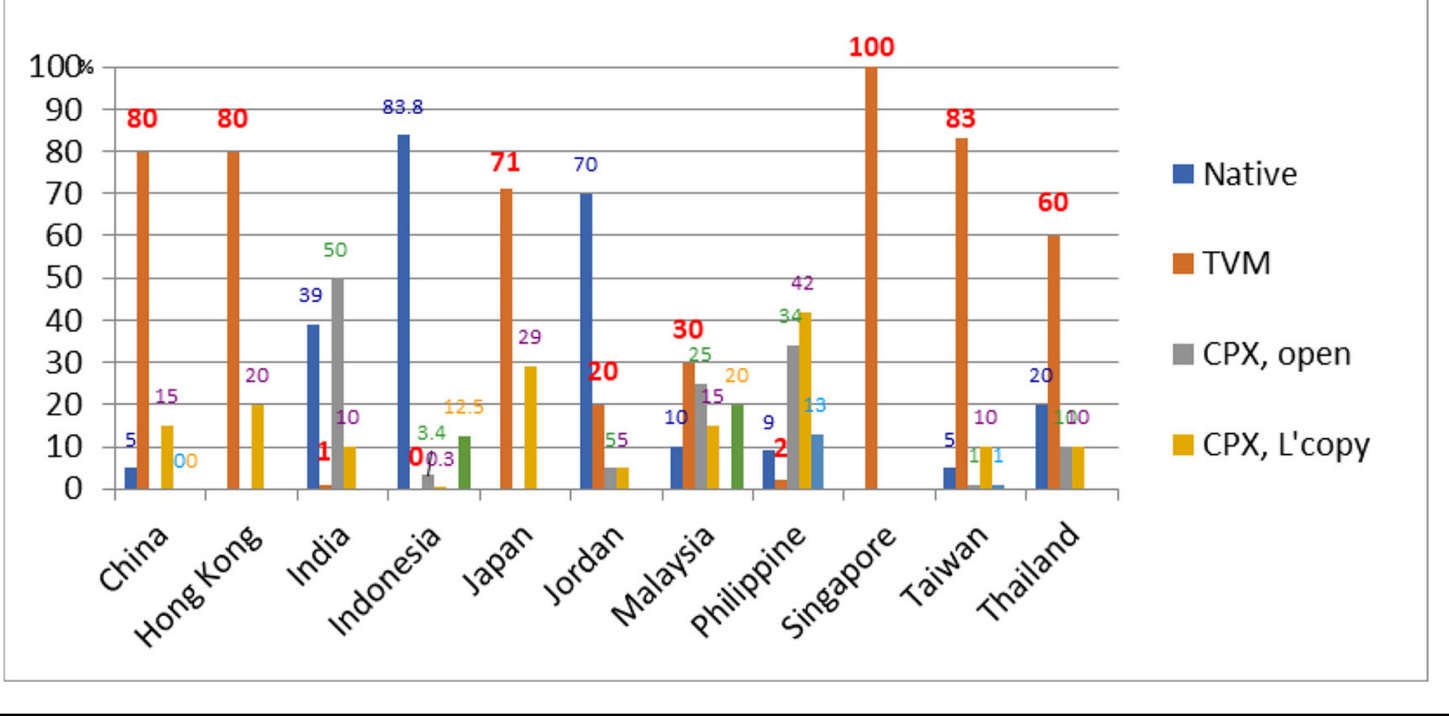

Fig. 3 Types of PRS for recurrent advanced prolapse in 11 selected countries/areas, Asia 2018

understand their effect. Ultimately, this is mostly to manage any complications arising when there are 'red flag' symptoms such as new-onset vaginal bleeding [3].

Authors' contribution TS Lo: Project development, Data collection, Data analysis, Manuscript editing.

YL Tan: Manuscript writing.

\section{Compliance with ethical standards}

Conflict of interest None.

\section{References}

1. FDA (2011) Uorgynecologic Surgical Mesh: Update on the Safety and Effectiveness of Transvaginal Placement for Pelvic Organ Prolapse. Center for Devices and Radiological Health.

2. Lo TS, Horng SG, Huang HJ, Lee SJ, Liang CC. Repair of recurrent vaginal vault prolapse using sacrospinous ligament fixation with mesh interposition and reinforcement. Acta Obstet Gynecol Scand. 2005;84:992-5.

3. Lo TS, Tan YL, Cortes EF, Wu PY, Pue LB, Al-Kharabsheh A. Clinical outcomes of mesh exposure/extrusion: presentation, timing and management. Aust N Z J Obstet Gynaecol. 2015;55(3):284-90.

Publisher's note Springer Nature remains neutral with regard to jurisdictional claims in published maps and institutional affiliations. 